\title{
Stability of the Calabi flow near an extremal metric
}

\author{
HONGNIAN HUANG AND KAI ZHENG
}

\begin{abstract}
We prove that on a Kähler manifold admitting an extremal metric $\omega$ and for any Kähler potential $\varphi_{0}$ close to $\omega$, the Calabi flow starting at $\varphi_{0}$ exists for all time and the modified Calabi flow starting at $\varphi_{0}$ will always be close to $\omega$. Furthermore, when the initial data is invariant under the maximal compact subgroup of the identity component of the reduced automorphism group, the modified Calabi flow converges to an extremal metric near $\omega$ exponentially fast.
\end{abstract}

Mathematics Subject Classification (2010): 53C44 (primary); 32Q15, 32Q26 (secondary).

\section{Introduction}

Let $M$ be a Kähler manifold and $\Omega$ be the Kähler class in $H^{2}(M, R) \cap H^{1,1}(M, C)$. By the $\partial \bar{\partial}$-lemma, any Kähler metric $\omega_{\varphi}$ in $\Omega$ can be written as

$$
\omega_{\varphi}=\omega+\sqrt{-1} \partial \bar{\partial} \varphi
$$

for some smooth real-valued Kähler potential $\varphi$. The space of Kähler metrics is defined by

$$
\mathcal{H}=\left\{\varphi \in C^{\infty}(M, R) \mid \omega+\sqrt{-1} \partial \bar{\partial} \varphi>0\right\} .
$$

Donaldson [17], Mabuchi [20] and Semmes [21] independently defined a WeilPeterson type-metric on $\mathcal{H}$, under which $\mathcal{H}$ becomes a non-positively curved infinite dimensional symmetric space. Chen [5] proved that any two points in $\mathcal{H}$ can be connected by a $C^{1,1}$ geodesic and that $\mathcal{H}$ is a metric space, which verifies two of Donalson's conjectures.

In order to tackle the existence of a constant scalar curvature Kähler metric (cscK) problem, Calabi [2,3] introduced a well-known functional

$$
C a(\varphi)=\int_{M} S(\varphi)^{2} \omega_{\varphi}^{n},
$$

Received June 3, 2010; accepted in revised form November 9, 2010. 
where $S(\varphi)$ is the scalar curvature of $\omega_{\varphi}$. The critical point of this Calabi functional is called an extremal Kähler metric. Calabi discovered that an extremal Kähler metric is a cscK if and only if the Calabi-Futaki invariant is equal to zero. Later, he suggested that one may study the gradient flow of the K-energy to search for the cscK. This flow is defined as

$$
\frac{\partial \varphi}{\partial t}=S-\underline{S}
$$

and it decreases the Calabi energy. Since (1.1) is a fourth order equation, the maximal principle fails. In [18], Donaldson proposed a programme to study the convergence of the Calabi flow. On a Riemannian surface, P. Chruściel [16] proved that the flow exists for all time and converges to a cscK metric by using the Bondi mass. Later Chen [6] and Struwe [22] gave a different proof assuming the uniformization theorem. In Chen-Zhu [15], they removed the assumption of the uniformization theorem. For higher dimensions, the Calabi flow has been studied in Chen-He [8-10] and Tosatti-Weinkove [23]. In Chen-He [8], they proved that the Calabi flow can start from a $C^{3, \alpha}$ Kähler potential and become smooth immediately as $t>0$.

One defines the little Hölder space $c^{k, \alpha}$ to be the closure of smooth functions in the usual Hölder norm $C^{k, \alpha}$.

Theorem 1.1 ([8]). If $\omega_{\varphi_{0}}=\omega+\sqrt{-1} \partial \bar{\partial} \varphi_{0}$ satisfies $\left|\varphi_{0}\right|_{c^{3, \alpha}(M, g)} \leq K$, and $\lambda \omega<\omega_{0}=\omega_{\varphi_{0}}<\Lambda \omega$, where $K, \lambda, \Lambda$ are positive constants, then the Calabi flow initiating from $\varphi_{0}$ admits a unique solution

$$
\varphi(t) \in C\left([0, T], c^{3, \alpha}(M, g)\right) \cap C\left((0, T], c^{4, \alpha}(M, g)\right)
$$

for small $T=T(\lambda, \Lambda, K, \omega)$. More specifically, for any $t \in(0, T]$, there is a constant $C=C(\lambda, \Lambda, K, \omega)$ such that

$$
t^{1 / 4}\left(|\dot{\varphi}(t)|_{c^{0, \alpha}}(M)+|\varphi(t)|_{c^{4, \alpha}(M)}\right) \leq C,|\varphi(t)|_{c^{3, \alpha}(M)} \leq C .
$$

Remark 1.2. He [19] shows that the Calabi flow can start from $\omega_{\varphi}$ where $\varphi \in$ $c^{2, \alpha}(M)$.

Theorem 1.3 ([8]). The solution obtained above belongs to

$$
C^{0}\left([0, T], c^{3, \alpha}(M)\right) \cap C^{0}\left((0, T], C^{\infty}(M)\right) .
$$

Chen and He then use an energy argument to show that when Kähler manifolds admits a $\operatorname{cscK} \omega$ and the initial Kähler potential is $C^{3, \alpha}$ small, the Calabi flow converges exponentially fast to a cscK nearby. In He [19], he improved this result for $C^{2, \alpha}$ small initial Kähler potentials.

In this short note, we prove a parallel theorem for extremal Kähler metrics using a different method from Chen-Ding-Zheng [7]. In that paper, they defined a flow called the pseudo-Calabi flow. They proved the short time existence from $c^{2, \alpha}$ initial Kähler potentials, the long time existence under uniform Ricci bounds 
and the stability near a cscK. Since the linearized operator of the pseudo-Calabi flow is not self-adjoint, they set up a unified frame to tackle the stability problem of the Kähler Ricci flow ( $c f$. Zheng [24]), the Calabi flow and the pseudo-Calabi flow. This method strongly relies on the geometric structure of the space of Kähler metrics.

First, we will prove the long time existence of the Calabi flow.

Theorem 1.4. On Kähler manifolds admitting an extremal metric $\omega$ and for any positive constant $\mathcal{K}, \lambda$, there is a small constant $\epsilon$ depending on $\omega, \mathcal{K}, \lambda$, such that for any Kähler potential $\varphi_{0}$, if

$$
\left|\varphi_{0}\right|_{C^{2, \alpha}(M)}<\mathcal{K}, \quad \lambda \omega<\omega_{\varphi_{0}}, \quad \int_{M}\left|\varphi_{0}\right|^{2} \omega^{n}<\epsilon,
$$

then the Calabi flow exists for all time.

Next we want to study the modified Calabi flow. Let $K$ be a maximal compact subgroup in the reduced automorphism group. Denote the corresponding Lie algebra of $K$ by $h_{0}(M)$, which is the ideal of holomorphic vector fields with zeros. For any holomorphic vector field $\tilde{Y} \in h_{0}(M)$, denote $\tilde{Y}=Y-\sqrt{-1} J Y$. Then there is a real function $\theta_{Y}$ such that

$$
L_{\tilde{Y}} \omega=L_{Y} \omega=\sqrt{-1} \partial \bar{\partial} \theta_{Y}(t)
$$

and

$$
\int_{M} \theta_{Y} \omega^{n}=0
$$

For an arbitrary metric

$$
\omega_{\varphi}=\omega+\sqrt{-1} \partial \bar{\partial} \varphi
$$

the corresponding $\theta_{\tilde{Y}}(\varphi)$ is

$$
\theta_{\tilde{Y}}(\varphi)=\theta_{Y}+\tilde{Y}(\varphi)
$$

Following Futaki-Mabuchi [1], suppose $\tilde{X}, \tilde{Y} \in h_{0}(M)$, then the bilinear form

$$
B(\tilde{X}, \tilde{Y})=\int_{M} \theta_{\tilde{X}(\varphi)} \theta_{\tilde{Y}(\varphi)} \omega_{\varphi}^{n}
$$

is independent of the choice of $\omega_{\varphi}$ in the Kähler class $\Omega=[\omega]$.

Let $\varphi(t)$ be a one parameter of Kähler potentials satisfying the Calabi flow equation and let $\sigma(t)$ be the holomorphic group generated by $X$, the real part of the extremal vector field $\tilde{X}$. Then $\sigma^{*}(t) \omega=\omega+i \partial \bar{\partial} \rho(t)$ satisfies the Calabi flow equation since

$$
\frac{\partial \sigma^{*}(t) \omega}{\partial t}=L_{X} \omega(t)=i \partial \bar{\partial}(S(t)-\underline{S})
$$


Hence we can choose $\rho(t)$ to be a parameter of Kähler potentials satisfying the Calabi flow equation starting from 0 .

Let $\psi(t)=\sigma(-t)^{*}(\varphi(t)-\rho(t))$. Notice that, by definition, $X=\sigma_{*}^{-1}\left(\frac{\partial}{\partial t} \sigma\right)$. So we obtain the modified Calabi flow,

$$
\begin{aligned}
\frac{\partial \psi}{\partial t} & =-X(\psi(t))+\sigma(-t)^{*}\left(\frac{\partial \varphi(t)}{\partial t}-\frac{\partial \rho(t)}{\partial t}\right) \\
& =-X(\psi(t))+\sigma(-t)^{*}(S(\varphi(t))-\underline{S})-(S(\omega)-\underline{S}) \\
& =S_{\psi}-\underline{S}-\theta_{X}-X(\psi) \\
& =S_{\psi}-\underline{S}-\theta_{X}(\psi) .
\end{aligned}
$$

Theorem 1.5. On Kähler manifolds admitting an extremal metric $\omega$, for any $\mathcal{K}$ invariant Kähler potential $\varphi_{0}$ close to $\omega$ (in the sense of Theorem (1.4)), the modified Calabi flow exponentially converges to a nearby extremal metric.

ACKnowledgements. The authors want to thank Professor X. X. Chen for many useful discussions on the stability problem of Calabi flow. They also want to express their gratitude to Professor V. Apostolov for his interest and help in this problem. The second author wants to thank his advisor Professor W. Y. Ding, also Professor X. H. Zhu and Professor H. Z. Li for their help and encouragement. The first author would like to thank Professor P. F. Guan for valuable discussions.

\section{Long time existence}

First of all, we would like to give a rough estimate of the geodesic distance between any two Kähler potentials $\varphi_{0}, \varphi_{1}$ when $\omega_{\varphi_{0}}, \omega_{\varphi_{1}}<\Lambda \omega$.

Lemma 2.1. $d\left(\varphi_{0}, \varphi_{1}\right)<C(\Lambda)\left(\int_{M}\left|\varphi_{0}-\varphi_{1}\right|^{2} \omega^{n}\right)^{\frac{1}{2}}$.

Proof. Let $\varphi_{t}=(1-t) \varphi_{0}+t \varphi_{1}$ for $0 \leq t \leq 1$. Then

$$
\begin{aligned}
d\left(\varphi_{0}, \varphi_{1}\right) & \leq L\left(\gamma_{t}\right)=\int_{0}^{1}\left(\int_{M}\left(\frac{\partial \gamma_{t}}{\partial t}\right)^{2} \omega_{\gamma_{t}}^{n}\right)^{\frac{1}{2}} d t \\
& \leq \int_{0}^{1}\left(\int_{M}\left(\varphi_{0}-\varphi_{1}\right)^{2} \omega_{\gamma_{t}}^{n}\right)^{\frac{1}{2}} d t \\
& \leq C(\Lambda)\left(\int_{M}\left|\varphi_{0}-\varphi_{1}\right|^{2} \omega^{n}\right)^{\frac{1}{2}} .
\end{aligned}
$$

We are ready to give a proof of Theorem 1.4. 
Proof. Suppose that the conclusion fails, then there exist positive constants $\mathcal{K}, \lambda, \Lambda$ and a sequence of $\varphi_{s}^{0}$ such that

$$
\left|\varphi_{s}^{0}\right|_{C^{2, \alpha}}<\mathcal{K}, \lambda \omega<\omega_{\varphi_{0}^{s}}<\Lambda \omega, \int_{M}\left|\varphi_{s}^{0}\right|^{2} \omega^{n}<\frac{1}{s} \quad s=1,2,3 \cdots .
$$

By virtue of the short time existence theorem, we get a sequence of solutions $\varphi_{S}(t)$ satisfying the flow equation $(1.1)$ with $\varphi_{s}(0)=\varphi_{s}^{0}$. Let $T_{s}$ be the first time such that

$$
\left|\varphi_{s}\left(T_{s}\right)-\rho\left(T_{s}\right)\right|_{c^{2, \alpha}\left(\omega\left(T_{s}\right)\right)}=2 C \text { holds }
$$

or

$$
\lambda \omega\left(T_{s}\right)<\omega_{\varphi_{s}\left(T_{s}\right)}<\Lambda \omega\left(T_{s}\right) \quad \text { fails, }
$$

where the constant $C$ is from Theorem 1.1. Then $T_{s}$ is bounded from below for sufficiently large $s$. Otherwise there is a subsequence of $\varphi_{S}\left(T_{S}\right)$ converging to $\varphi_{\infty}$ in the $C^{2, \alpha^{\prime}}(\omega)$ sense, where $\alpha^{\prime}<\alpha$. Notice that $\lambda \omega \leq \omega_{\varphi_{\infty}} \leq \Lambda \omega$, but that $\lambda \omega<\omega_{\varphi_{\infty}}<\Lambda \omega$ fails.

On the other hand, Lemma 2.1 shows that $d\left(0, \varphi_{s}^{0}\right) \rightarrow 0$ as $s \rightarrow \infty$. Since the distance function decreases under the Calabi flow, we have

$$
d\left(\rho\left(T_{S}\right), \varphi_{s}\left(T_{S}\right)\right) \rightarrow 0
$$

as $s \rightarrow \infty$. Let $\varphi_{\infty}(t)$ be one parameter potentials satisfying the Calabi flow equation initiating from $\varphi_{\infty}$. Then for $t_{0} \geq t$,

$$
\begin{aligned}
d\left(\rho\left(t_{0}\right), \varphi_{\infty}\left(t_{0}\right)\right) & \leq d\left(\rho(t), \varphi_{\infty}(t)\right) \\
& \leq d\left(\rho(t), \rho\left(T_{s}\right)\right)+d\left(\rho\left(T_{s}\right), \varphi_{s}\left(T_{s}\right)\right)+d\left(\varphi_{s}\left(T_{s}\right), \varphi_{\infty}(t)\right) .
\end{aligned}
$$

By Lemma 2.1, $d\left(\varphi_{s}\left(T_{s}\right), \varphi_{\infty}(t)\right) \rightarrow 0$ as $s \rightarrow \infty$ and $t \rightarrow 0$. Hence $\rho\left(t_{0}\right)=$ $\varphi_{\infty}\left(t_{0}\right)$, which implies $0=\varphi_{\infty}$, a contradiction.

Moreover, from Theorem 1.3, we obtain the higher order uniform bounds of the sequence of the solutions:

$$
\left|\varphi_{s}\left(T_{s}\right)-\rho\left(T_{s}\right)\right|_{C^{k, \alpha}\left(\omega\left(T_{s}\right)\right)} \leq C(k), \forall k \geq 0 .
$$

Therefore we can choose a subsequence of $\phi_{S}=\sigma\left(-T_{S}\right)^{*}\left(\varphi_{S}\left(T_{S}\right)-\rho\left(T_{S}\right)\right)$ so that

$$
\phi_{s} \rightarrow \phi_{\infty} \text { in } C^{k, \alpha}(\omega), \forall k \geq 0,
$$

and

$$
\left|\phi_{\infty}\right|_{C^{2, \alpha}(\omega)}=2 C \quad\left(\text { or } \quad \lambda \omega<\omega_{\phi_{\infty}}<\Lambda \omega \text { fails }\right) .
$$

However, this contradicts the fact that $d\left(0, \phi_{\infty}\right)=0$.

Corollary 2.2. Given a Kähler potential $\varphi_{0}$ close to 0 in the sense of Theorem 1.4, then the modified Calabi flow stays in a neighborhood of 0 . If $\varphi_{0}$ is $\mathcal{K}$-invariant, then the modified Calabi flow converges to an extremal metric nearby. 
Proof. That the modified Calabi flow stays in a neighborhood of 0 can be easily seen from the regularity Theorem 1.3. Notice that the Calabi flow decreases the Calabi energy, i.e.

$$
\frac{\partial}{\partial t} C a\left(\omega_{\varphi}\right)=-2 \int_{M} \mathcal{L}_{\varphi}\left(S_{\varphi}\right) S_{\varphi} \omega_{\varphi}^{n},
$$

where $\mathcal{L}_{\varphi}$ is the Lichnerowicz operator with respect to $\omega_{\varphi}$. It follows that we can take a sequence of $t_{j} \rightarrow \infty$ such that

$$
\lim _{j \rightarrow \infty} \int_{M} \mathcal{L}_{\varphi\left(t_{j}\right)}\left(S_{\varphi\left(t_{j}\right)}\right) S_{\varphi\left(t_{j}\right)} \omega_{\varphi\left(t_{j}\right)}^{n}=0 .
$$

Then there is a subsequence of $t_{j}$ such that $\psi\left(t_{j}\right)$ converges to a potential $\psi_{\infty}$ in $C^{\infty}$ and

$$
\int_{M} \mathcal{L}_{\psi_{\infty}}\left(S_{\psi_{\infty}}\right) S_{\psi_{\infty}} \omega_{\psi_{\infty}}^{n}=0
$$

Hence $\omega_{\psi_{\infty}}$ is an extremal metric. If $\varphi_{0}$ is $\mathcal{K}$-invariant, then $\psi_{\infty}$ is a fixed point under the modified Calabi flow and the modified Calabi flow decreases the geodesic distance between $\psi(t)$ and $\psi_{\infty}$. Hence the flow converges to $\psi_{\infty}$.

\section{Exponential decay}

We define the modified Calabi energy as

$$
\widetilde{C a}(\psi)=\int_{M}\left(S(\psi)-\underline{S}-\theta_{X}(\psi)\right)^{2} \omega_{\psi}^{n} .
$$

The evolution of the modified Calabi energy along the modified Calabi flow is

$$
\begin{aligned}
\partial_{t} \int_{M} \dot{\psi}^{2} \omega_{\psi}^{n} & =\int_{M}\left(2 \dot{\psi} \ddot{\psi}+\dot{\psi}^{2} \triangle_{\psi} \dot{\psi}\right) \omega_{\psi}^{n} \\
& =2 \int_{M} \dot{\psi}\left(\dot{S}_{\psi}-\dot{\theta}_{X}(t)-\dot{\psi}_{i} \dot{\psi}^{i}\right) \omega_{\psi}^{n} \\
& =2 \int_{M} \dot{\psi}\left(-L \dot{\psi}+\dot{\psi}^{i} S_{i}-\dot{\theta}_{X}(t)-\dot{\psi}_{i} \dot{\psi}^{i}\right) \omega_{\psi}^{n} \\
& =2 \int_{M} \dot{\psi}\left(-L \dot{\psi}+\dot{\psi}^{i}\left(\dot{\psi}_{i}+\theta_{X}(t)_{i}\right)-\dot{\theta}_{X}(t)-\dot{\psi}_{i} \dot{\psi}^{i}\right) \omega_{\psi}^{n} \\
& =2 \int_{M} \dot{\psi}\left(-L \dot{\psi}+\dot{\psi}^{i} \dot{\psi}_{i}+X(\dot{\psi})-X(\dot{\psi})-\dot{\psi}_{i} \dot{\psi}^{i}\right) \omega_{\psi}^{n} \\
& =-2 \int_{M} \dot{\psi} L \dot{\psi} \omega_{\psi}^{n} .
\end{aligned}
$$

In this computation we use the identities

$$
\dot{\psi}^{i}\left(\theta_{X i}+(X(\psi))_{i}\right)=\dot{\psi}^{i}\left(\theta_{X}(t)\right)_{i}=X(\dot{\psi}) .
$$


The modified Calabi-Futaki invariant

$$
\tilde{F}(\tilde{Y})=F(\tilde{Y})-B(\tilde{X}, \tilde{Y})=\int_{M} \theta_{\tilde{Y}}(\psi)\left(S-\underline{S}-\theta_{\tilde{X}}(\psi)\right) \omega_{\psi}^{n}
$$

is equal to zero when $M$ admits an extremal metric $\omega$. This shows that the direction of the modified Calabi flow is always perpendicular to the kernel of the Lichnerowicz operator. To obtain exponential convergence, one needs to give a uniform lower bound of the the first eigenvalue of $L_{t}$ along the modified Calabi flow. More precisely, we have the following lemma which is similar to Chen-Li-Wang [11].

Lemma 3.1. Along the modified Calabi flow, there is a positive constant $\lambda>0$ such that for sufficiently large $t$ and for any

$$
f \in A_{t}=\left\{f \in C_{R}^{\infty}(M) \mid \int_{M} f \omega_{\psi(t)}^{n}=0 \text { and } \int_{M} \theta_{Y}(t) f \omega_{\psi(t)}^{n}=0, \forall \tilde{Y} \in h_{0}(M)\right\},
$$

we have

$$
\int_{M} L_{t}(f) f \omega_{\psi(t)}^{n} \geq \lambda \int_{M} f^{2} \omega_{\psi(t)}^{n} .
$$

Proof. If not, there must be a sequence $\psi_{s}=\psi(s)$ and $f_{s}$ such that

$$
\int_{M}\left|\left(f_{s}\right)_{i j}\right|^{2} \omega_{\psi_{s}}^{n}<\frac{1}{s} ; \int_{M} f_{s}^{2} \omega_{\psi_{s}}^{n}=1 ; \int_{M} f_{s} \omega_{\psi_{s}}^{n}=0 .
$$

Since the $C^{l}$ norm of $\psi_{s}$ is uniformly bounded for any $l \geq 0$. Using the Ricci identity

$$
\left.\int_{M}\left|\left(f_{s}\right)_{i}\right|^{2}\right|^{n} \psi_{s}=\int_{M}\left|\left(f_{s}\right)_{i j}\right|^{2} \omega_{\psi_{s}}^{n}+\int_{M} R^{i \bar{j}}\left(f_{s}\right)_{i}\left(f_{s}\right)_{\bar{j}} \omega_{\psi_{s}}^{n}
$$

and the interpolation inequality, we conclude that $f_{s}$ are uniformly $W^{2,2}$ bounded. So we can pass to the limit and get

$$
\int_{M}\left|\left(f_{\infty}\right)_{i j}\right|^{2} \omega_{\psi_{\infty}}^{n}=0 ; \int_{M} f_{\infty}^{2} \omega_{\psi_{\infty}}^{n}=1 ; \int_{M} f_{\infty} \omega_{\psi_{\infty}}^{n}=0 .
$$

Since in local coordinates, $\uparrow \bar{\partial} f_{\infty}$ is holomorphic in the weak sense, $f_{\infty}$ is smooth indeed. From the assumption of $A_{t}$ we have

$$
\int_{M} \theta_{Y}\left(\psi_{\infty}\right) f_{\infty} \omega_{\psi_{\infty}}^{n}=0, \forall \tilde{Y} \in h_{0}(M) .
$$

In particular, we may choose $\tilde{Y}=\uparrow \bar{\partial} f_{\infty} \in h_{0}(M)$. Hence,

$$
\int_{M} f_{\infty}^{2} \omega_{\infty}^{n}=0
$$

This contradicts (3.3). 
It is easy to see that $\widetilde{C a}(\psi(t)) \leq C e^{-\lambda t}$. To get exponential convergence of $\psi(t)$, we calculate the evolution formula for $\int_{M}\left|\nabla^{k}\left(\psi(t)-\psi_{\infty}\right)\right|^{2} \omega^{n}$ :

$$
\begin{aligned}
& \frac{\partial}{\partial t} \int_{M}\left|\nabla^{k}\left(\psi(t)-\psi_{\infty}\right)\right|^{2} \omega^{n} \\
= & \int_{M} \nabla^{k}\left(S-\underline{S}-\theta_{X}(\psi)\right) * \nabla^{k}\left(\psi(t)-\psi_{\infty}\right) \omega^{n} \\
= & \int_{M}\left(S-\underline{S}-\theta_{X}(\psi)\right) * \nabla^{2 k}\left(\psi(t)-\psi_{\infty}\right) \omega^{n} \\
\leq & \left(\int_{M}(S-\underline{S}-\theta(X))^{2} \omega^{n}\right)^{1 / 2}\left(\int_{M}\left|\nabla^{2 k}\left(\psi(t)-\psi_{\infty}\right)\right|^{2} \omega^{n}\right)^{1 / 2} \\
\leq & C\|S-\underline{S}-\theta(X)\|_{L^{2}(\omega)} \\
\leq & C\|S-\underline{S}-\theta(X)\|_{L^{2}\left(\omega_{t}\right)} \\
\leq & C e^{-\lambda_{2} t} .
\end{aligned}
$$

By the Sobolev embedding, we conclude that

$$
\left\|\psi_{t}-\psi_{\infty}\right\|_{C^{l}(\omega)} \leq\left\|\psi_{t}-\psi_{\infty}\right\|_{W^{k, 2}(\omega)} \leq C e^{-\lambda_{2} t}
$$

Hence we obtain the result stated in Theorem 1.5.

\section{References}

[1] A. FUTAKI and T. MABUCHI, Bilinear forms and extremal Kähler vector fields associated with Kähler classes, Math. Ann. 301 (1995), 199-210.

[2] E. CAlABI, Extremal Kähler metrics, In: "Seminar on Differential Geometry", Ann. of Math. Stud., Vol. 102, Princeton Univ. Press, Princeton, N.J., 1982, 259-290.

[3] E. CALABI, Extremal Kähler metrics. II, In: "Differential Geometry and Complex Analysis", Springer, Berlin, 1982, 95-114.

[4] E. Calabi and X. X. Chen, The space of Kähler metrics. II, J. Differential Geom. 61 (2002), 173-193.

[5] X. X. ChEn, The space of Kähler metrics, J. Differential Geom. 56 (2000), 189-234.

[6] X. X. Chen, Calabi flow in Riemann surfaces revisited: a new point of view, Internat. Math. Res. Notices 2001, 275-297.

[7] X. X. Chen, W. Y. Ding and K. Zheng, Pseudo-Calabi flow, Unpublished, 2009.

[8] X. X. Chen and W. Y. He, On the Calabi flow, Amer. J. Math. 130 (2008), 539-570.

[9] X. X. CHEN and W. Y. HE, The Calabi flow on Kähler surface with bounded Sobolev constant, (I), arXiv:0710.5159, 2007.

[10] X. X. Chen and W. Y. He, The Calabi flow on toric Fano surface, arXiv:0807.3984, 2008.

[11] X. X. Chen, H. Z. LI and B. WANG, Kähler-Ricci flow with small initial energy, Geom. Funct. Anal. 18 (2009), 1525-1563.

[12] X. X. Chen and G. Tian, Ricci flow on Kähler manifolds, C. R. Acad. Sci. Paris Sér. I Math. 332 (2001), 245-248.

[13] X. X. Chen and G. Tian, Ricci flow on Kähler-Einstein surfaces, Invent. Math. 147 (2002), 487-544. 
[14] X. X. Chen and G. Tian, Uniqueness of extremal Kähler metrics, C. R. Math. Acad. Sci. Paris 340 (2005), 287-290.

[15] X. X. CHEN and M. J. ZHU, Liouville energy on a topological two sphere, arXiv:0710.4320, 2007.

[16] P. T. ChruśCIEL, Semi-global existence and convergence of solutions of the RobinsonTrautman (2-dimensional Calabi) equation, Comm. Math. Phys. 137 (1991), 289-313.

[17] S. K. Donaldson, Symmetric spaces, Kähler geometry and Hamiltonian dynamics, In: "Northern California Symplectic Geometry Seminar", Amer. Math. Soc. Transl. Ser. 2, 196, Amer. Math. Soc., Providence, RI, 1999, 3-33.

[18] S. K. Donaldson, Conjectures in Kähler geometry, In: "Strings and Geometry", Amer. Math. Soc., Providence, RI, 2004, 71-78.

[19] W. Y. HE, Local solution and extension to the Calabi flow, arXiv:0904.0978, 2009.

[20] T. MABUCHI, Some symplectic geometry on compact Kähler manifolds. I, Osaka J. Math. 24 (1987), 227-252.

[21] S. Semmes, Complex Monge-Ampère and symplectic manifolds, Amer. J. Math. 114 (1992), 495-550.

[22] M. StRuwe, Curvature flows on surfaces, Ann. Scuola Norm. Sup. Pisa Cl. Sci. (5) (2002), 247-274.

[23] V. Tos atti and B. Weinkove, The Calabi flow with small initial energy, Math. Res. Lett. 14 (2007), 1033-1039.

[24] K. ZHENG, Stability of the Kähler Ricci flow in the space of Kähler metrics, Pacific J. Math. 251 (2011), 469-497.

Centre interuniversitaire de recherches

en géométrie et topologie

Université du Québec à Montréal

Case postale 8888, Succursale centre-ville

Montréal (Québec), H3C 3P8, Canada

hnhuang@gmail.com

Academy of Mathematics and Systems Sciences

Chinese Academy of Sciences

Beijing, 100190, P.R. China

kaizheng@amss.ac.cn 